\section{Predicting Exchange Rate Volatility: Genetic Programming Versus GARCH and RiskMetrics $^{\mathrm{TM}}$}

\author{
Christopher J. Neely and Paul A. Weller
}

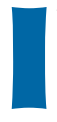
$\mathrm{t}$ is well established that the volatility of asset prices displays considerable persistence. That is, large movements in prices tend to be followed by more large moves, producing positive serial correlation in squared returns. Thus, current and past volatility can be used to predict future volatility. This fact is important to both financial market practitioners and regulators.

Professional traders in equity and foreign exchange markets must pay attention not only to the expected return from their trading activity but also to the risk that they incur. Risk-averse investors will wish to reduce their exposure during periods of high volatility, and improvements in risk-adjusted performance depend upon the accuracy of volatility predictions. Many current models of risk management, such as Value-at-Risk (VaR), use volatility predictions as inputs.

The bank capital adequacy standards recently proposed by the Basel Committee on Banking Supervision illustrate the importance of sophisticated risk management techniques for regulators. These norms are aimed at providing international banks with greater incentives to manage financial risk in a sophisticated fashion, so that they might economize on capital. One such system that is widely used is RiskMetrics ${ }^{\mathrm{TM}}$, developed by J.P. Morgan.

A core component of the RiskMetrics system is a statistical model-a member of the large ARCH/ GARCH family - that forecasts volatility. Such ARCH/

Christopher J. Neely is a research officer at the Federal Reserve Bank of St. Louis. Paul A. Weller is a professor of finance at the Henry B. Tippie College of Business Administration of the University of Iowa This paper is a revised and expanded version of a chapter entitled "Using a Genetic Program to Predict Exchange Rate Volatility," in Genetic Algorithms and Genetic Programming in Computational Finance, edited by Shu-Heng Chen, published by Kluwer Academic Publishers. We would like to thank Janis Zvingelis for excellent programming assistance. Charles Hokayem provided research assistance.

(1) 2002, The Federal Reserve Bank of St. Louis.
GARCH models are parametric. That is, they make specific assumptions about the functional form of the data generation process and the distribution of error terms. Parametric models such as GARCH are easy to estimate and readily interpretable, but these advantages may come at a cost. Other, perhaps much more complex models may be better representations of the underlying data generation process. If so, then procedures designed to identify these alternative models have an obvious payoff. Such procedures are described as nonparametric. Instead of specifying a particular functional form for the data generation process and making distributional assumptions about the error terms, a nonparametric procedure will search for the best fit over a large set of alternative functional forms.

This article investigates the performance of a genetic program applied to the problem of forecasting volatility in the foreign exchange market. Genetic programming is a computer search and problemsolving methodology that can be adapted for use in nonparametric estimation. It has been shown to detect patterns in the conditional mean of foreign exchange and equity returns that are not accounted for by standard statistical models (Neely, Weller, and Dittmar, 1997; Neely and Weller, 1999, 2001; Neely, 2001). These achievements suggest that a genetic program may also be a powerful tool for generating predictions of asset price volatility.

We compare the performance of a genetic program in forecasting daily exchange rate volatility for the dollar-Deutsche mark and dollar-yen exchange rates with that of a GARCH( 1,1$)$ model and a related RiskMetrics volatility forecast (described in the following section). These models are widely used by both academics and practitioners and thus are good benchmarks with which to compare the genetic program forecasts. While the overall forecast performance of the two methods is broadly similar, on some dimensions the genetic program produces significantly superior results. This encouraging finding suggests that more detailed investigation of this methodology applied to volatility forecasting would be warranted.

\section{THE BENCHMARK MODEL}

Before discussing the genetic programming procedure, we will review the benchmark GARCH and RiskMetrics volatility models. Engle (1982) developed the autoregressive conditionally heteroskedastic $(\mathrm{ARCH})$ model to characterize the observed serial correlation in asset price volatility. Suppose 
we assume that a price $P_{t}$ follows a random walk,

$$
P_{t+1}=P_{t}+\varepsilon_{t+1},
$$

where $\varepsilon_{t+1} \sim N\left(0, \sigma_{t}^{2}\right)$. The variance of the error term depends upon $t$, and the objective of the model is to characterize the way in which this variance changes over time. The ARCH model assumes that this dependence can be captured by an autoregressive process of the form

$$
\sigma_{t}^{2}=\omega+\alpha_{0} \varepsilon_{t}^{2}+\alpha_{1} \varepsilon_{t-1}^{2}+\cdots+\alpha_{m} \varepsilon_{t-m}^{2},
$$

where the restrictions $\omega \geq 0$ and $\alpha_{i} \geq 0$ for $i=0,1, \ldots, m$ ensure that the predicted variance is always nonnegative. This specification illustrates clearly how current levels of volatility will be influenced by the past and how periods of high or low price fluctuation will tend to persist.

Bollerslev (1986) extended the ARCH class to produce the generalized autoregressive conditionally heteroskedastic (GARCH) model, in which the variance is given by

$$
\begin{aligned}
\sigma_{t}^{2} & =\omega+\beta_{1} \sigma_{t-1}^{2}+\beta_{2} \sigma_{t-2}^{2}+\cdots+\beta_{k} \sigma_{t-k}^{2} \\
& +\alpha_{0} \varepsilon_{t}^{2}+\alpha_{1} \varepsilon_{t-1}^{2}+\cdots+\alpha_{m} \varepsilon_{t-m}^{2} .
\end{aligned}
$$

The simplest specification in this class, and the one most widely used, is referred to as $\operatorname{GARCH}(1,1)$ and is given by

$$
\sigma_{t}^{2}=\omega+\beta \sigma_{t-1}^{2}+\alpha \varepsilon_{t}^{2} .
$$

When $\alpha+\beta<1$, the variance process displays mean reversion to the unconditional expectation of $\sigma_{t}^{2}$, $\omega /(1-\alpha-\beta)$. That is, forecasts of volatility in the distant future will be equal to the unconditional expectation of $\sigma_{t}^{2}, \omega /(1-\alpha-\beta)$.

The RiskMetrics model for volatility forecasting imposes the restrictions that $\alpha+\beta=1$ and that $\omega=0 .{ }^{1}$ In addition, the parameter $\beta$ is not estimated, but imposed to be equal to 0.94 (J.P. Morgan/Reuters, 1996). This value was found to minimize the meansquared error (MSE) of volatility forecasts for asset prices. The RiskMetrics one-day-ahead volatility forecast is

$$
\sigma_{t}^{2}=\beta \sigma_{t-1}^{2}+(1-\beta) \varepsilon_{t}^{2} .
$$

The GARCH model has been used to characterize patterns of volatility in U.S. dollar foreign exchange markets (Baillie and Bollerslev, 1989, 1991) and in the European Monetary System (Neely, 1999). However, initial investigations into the explanatory power of out-of-sample forecasts produced disappointing results (West and Cho, 1995). Jorion (1995) found that volatility forecasts for several major currencies from the GARCH model were outperformed by implied volatilities generated from the Black-Scholes option-pricing model. These studies typically used the squared daily return as the variable to be forecast. However, the squared return is a very imprecise measure of true, unobserved volatility. For example, the exchange rate may move around a lot during the day, and yet end up close to its value the same time the previous day. In this case, the squared daily return would be small, even though volatility was high. More recently, it has been demonstrated that one can significantly improve the forecasting power of the GARCH model by measuring volatility as the sum of intraday squared returns (Andersen and Bollerslev, 1998). This measure is referred to as integrated, or realized, volatility. In theory, if the true underlying price path is a diffusion process, it is possible to obtain progressively more accurate estimates of the true volatility by increasing the frequency of intraday observation. Of course, there are practical limits to this; microstructural effects begin to degrade accuracy beyond a certain point.

\section{GENETIC ALGORITHMS AND GENETIC PROGRAMMING}

Genetic algorithms are computer search procedures used to solve appropriately defined problems. The structure of the search procedure is based on the principles of natural selection. These procedures were developed for genetic algorithms by Holland (1975) and extended to genetic programming by Koza (1992). The essential features of both algorithms include (i) a means of representing potential solutions to a problem as character strings that can be split up and recombined to form new potential solutions and (ii) a fitness criterion that measures the "quality" of a candidate solution. Both types of algorithms produce successive "generations" of candidate solutions using procedures that mimic genetic reproduction and recombination. Each new generation is subjected to the pressures of "natural selection" by increasing the probability that candidate solutions scoring highly on the fitness criterion get to reproduce.

To understand the principles involved in genetic

\footnotetext{
1 The restriction, $\alpha+\beta=1$, implies that shocks to the volatility process persist forever; higher volatility today will lead one to forecast higher volatility indefinitely. It therefore falls into the class of integrated GARCH or IGARCH models.
} 
programming, it is useful to understand the operation of the simpler genetic algorithm. Genetic algorithms require that potential solutions be expressed as fixed-length character strings. Consider a problem in which candidate solutions are mapped into binary strings $s$, with a length of five digits. One possible solution would be represented as (01010). Associated with this binary string would be a measure of fitness that quantifies how well it solves the problem. In other words, we need a fitness function $m(s)$ that maps the strings into the real line and thus ranks the quality of the solutions. Next we introduce the crossover operator. Given two strings, a crossover point is randomly selected and the first part of one string is combined with the second part of the other. For example, given the two strings (00101) and (11010) and a crossover point between elements two and three, the new string (00010) is generated. The remaining parts of the original strings are discarded.

The algorithm begins by randomly generating an initial population of binary strings and then evaluating the fitness of each string by applying the fitness function $m(s)$. Next, the program produces a new (second) generation of candidate solutions by selecting pairs of strings at random from this initial population and applying the crossover operator to create new strings. The probability of selecting a given string is set to be proportional to its fitness. Thus a "selection pressure" in favor of progressively superior solutions is introduced. This process is repeated to produce successive generations of strings, keeping the size of each generation the same. The procedure "evolves" new generations of improved potential solutions.

Recall that genetic algorithms require that potential solutions be encoded as fixed length character strings. Koza's (1992) extension, genetic programming, instead employs variable-length, hierarchical strings that can be thought of as decision trees or computer programs. However, the basic structure of a genetic program is exactly the same as described above. In particular, the crossover operator is applied to pairs of decision trees to generate new "offspring" trees.

The application in this paper represents forecasting functions as trees and makes use of the following function set in constructing them: plus, minus, times, divide, norm, log, exponential, square root, and cumulative standard normal distribution function. In addition, we supply the following set of data functions: data, average, max, min, and lag. The data functions can operate on any of the four data series that we provide as inputs to the genetic program: (i) daily foreign exchange returns, (ii) integrated volatility (i.e., the sum of squared intraday returns), (iii) the sum of the absolute value of intraday returns, and (iv) the number of days until the next business day. For example, data(returns $(t))$ is simply the identity function that computes the daily return at $t$. The other data functions operate in a similar fashion, but also take numerical arguments to specify the length of the window-the number of observations-over which the functions operate. The numerical arguments that the functions take are determined by the genetic program. Thus average $($ returns $(t))(n)$ generates the arithmetic average of the return observations $t, t-1, \ldots, t-n+1$.

The choice of elements to include in the function set is a potentially important one. While a genetic program can, in principle, produce a very highly complex solution from simple functions, computational limitations might make such solutions very difficult to find in practice. Providing specialized functions to the genetic program that are thought to be useful to a "good" solution to the problem can greatly increase the efficiency of the search by encouraging the genetic program to search in the area of the solution space containing those functions. On the other hand, this might bias the genetic program's search away from other promising regions. To focus the search in promising regions of the solution space, we investigate the results of adding three additional complex data functions to the original set of functions. This is described below.

The expanded set of data functions consists of the original set plus geo, mem, and arch5. Each of these functions approximates the forecast of a known parametric model of conditional volatility. Thus, the genetic program might find them useful. The function geo returns the following weighted average of ten lags of past data:

$$
\operatorname{geo}(\operatorname{data})(\alpha) \equiv \sum_{j=0}^{9} \alpha\left[(1-\alpha)^{j}\right] \operatorname{data}_{t-j} .
$$

This function can be derived from the prediction of an IGARCH specification with parameter $\alpha$, where we constrain $\alpha$ to satisfy $0.01 \leq \alpha \leq 0.99$ and ten lags of data are used. The function mem returns a weighted sum similar to that which would be obtained from a long memory specification for volatility. It takes the form

$$
\operatorname{mem}(\text { data })(d) \equiv \sum_{j=0}^{9} h_{j} \text { data }_{t-j},
$$




\section{Figure 1}

\section{Example of a Hypothetical Forecast Function}

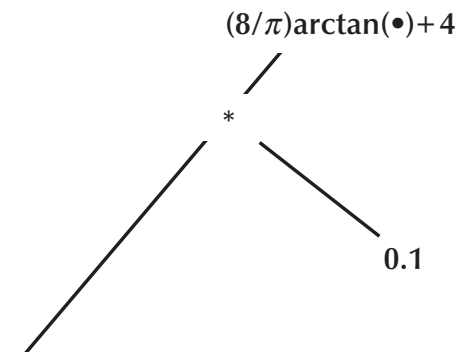

$\operatorname{Max}($ sum of squared intraday returns)(•)

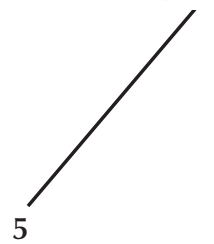

where $h_{0}=1, h_{j} \propto(1 / j !)(d+j-1)(d+j-2) \ldots(d+1) d$ for $j>0$ and the sum of the coefficients $h_{j}$ is constrained to equal 1 so that the output would be of the same magnitude as recent volatility. The parameter $d$ is determined by the genetic program and constrained to satisfy $-1<d<1$. Finally, the function arch 5 permits a flexible weighting of the five most recent observations, where the values for $h_{j}$ are provided by the genetic program and constrained to lie within $\{-5,5\}$ and to sum to 1 . Again, the constraint on the sum of the coefficients ensures that the magnitude of the output will be similar to that of recent volatility. The function has the form

(8) $\operatorname{arch} 5(\operatorname{data})(h) \equiv \sum_{j=0}^{4} h_{j} \operatorname{data}_{t-j}, \quad h=\left(h_{0}, h_{1}, \ldots, h_{4}\right)$.

Figure 1 illustrates a simple example of a hypothetical tree determining a forecasting function. The function first computes the maximum of the sum of squared intraday returns over the last five days. This number is multiplied by 0.1 , and the result is entered as the argument $x$ of the function $(8 / \pi) \arctan (x)+4$. This latter function is common to all trees and maps the real line into the interval $(0,8)$. It ensures that all forecasts are nonnegative and bounded above by a number chosen with reference to the characteristics of the in-sample period.

We now turn to the form of the fitness criterion. Because true volatility is not directly observed, it is necessary to use an appropriate proxy in order to assess the volatility forecasting performance of the genetic program. One possibility is to use the ex post squared daily return. However, as Andersen and Bollerslev (1998) have pointed out, this is an extremely noisy measure of the true underlying volatility and is largely responsible for the apparently poor forecast performance of GARCH models. A better approach is to sum intraday returns to measure true daily volatility (i.e., integrated volatility) more accurately. We measure integrated volatility using five irregularly spaced intraday observations. If $S_{i, t}$ is the $i$ th observation on date $t$, we define

$$
\begin{gathered}
R_{i, t}=100 \cdot \ln \left(\frac{S_{i+1, t}}{S_{i, t}}\right) \quad \text { for } i=1,2,3, \text { and } 4 \\
=100 \cdot \ln \left(\frac{S_{1, t+1}}{S_{5, t}}\right) \quad \text { for } i=5 \\
\sigma_{I, t}^{2}=\sum_{i=1}^{5} R_{i, t}^{2} .
\end{gathered}
$$

Thus $\sigma_{I, t}^{2}$ is the measure of integrated volatility on date $t .^{2}$ Using five intraday observations represents a compromise between the increase in accuracy generated by more frequent observations and the problems of data handling and availability that arise as one moves to progressively higher frequencies of intraday observation.

In constructing the rules, the genetic program minimized the mean-squared forecast error (MSE) as the fitness criterion. There are potential inefficiencies involved in using this criterion on heteroskedastic data. However, a heteroskedasticitycorrected fitness measure proved unsatisfactory in experiments. With three to five observations per day, there were instances where the integrated daily volatility was very small; the heteroskedasticity correction caused the measure to be inappropriately sensitive to those observations. ${ }^{3}$

\footnotetext{
2 More precisely, daily volatility is calculated from 1700 Greenwich Mean Time (GMT) to 1700 GMT.

3 A perennial problem with using flexible, powerful search procedures like genetic programming is overfitting - the finding of spurious patterns in the data. Given the well-documented tendency for the genetic program to overfit the data, it is necessary to design procedures to mitigate this (e.g., Neely, Weller, and Dittmar, 1997). Here, we investigated the effect of modifying the fitness criterion by adding a penalty for complexity. This penalty consisted of subtracting an amount $(0.002 \times$ number of nodes $)$ from the negative MSE. Nodes are data and numerical functions. This modification is intended to bias the search toward functions with fewer nodes, which are simpler and therefore less prone to overfit the data. Unfortunately, this procedure produced no significant changes in performance, so we will report results only from the unmodified version.
} 


\section{Table 1}

\section{Data Type and Source}

\begin{tabular}{lll} 
Time & \multicolumn{1}{c}{ Source } & \multicolumn{1}{c}{ Type of price } \\
\hline 1000 & Swiss National Bank & Triangular arbitrage on bid rates \\
1400 & Federal Reserve Bank of New York & Midpoint of bid and ask \\
1600 & Bank of England & Triangular arbitrage, unspecified \\
1700 & Federal Reserve Bank of New York & Midpoint of bid and ask \\
2200 & Federal Reserve Bank of New York & Midpoint of bid and ask \\
\hline
\end{tabular}

\section{DATA AND IMPLEMENTATION}

The object of this exercise is to forecast the daily volatility (the sum of intraday squared returns) of two currencies against the dollar, the German mark (DEM) and Japanese yen (JPY), over the period June 1975 to September 1999. Thus, the final nine months of data for the DEM represent the rate derived from that of the euro, which superseded the DEM in January 1999. The timing of observations was $1000,1400,1600,1700$, and 2200 GMT. Days with fewer than three valid observations or no observation at 1700 were treated as missing. In addition, weekends were excluded. The sources of the data for both exchange rates are summarized in Table 1. We provided the genetic program with three series in addition to the integrated volatility series: daily returns, sum of absolute intraday returns, and the number of days until the next trading day.

The full sample is divided into three subperiods: the training period June 1975 through December 1979; the selection period January 1980 through December 30, 1986; and the out-of-sample period December 31, 1986, through September 21, 1999. The role of these subperiods is described below.

In searching through the solution space of forecasting functions, the genetic program followed the procedures below.

1. Create an initial generation of 500 randomly generated forecast functions.

2. Measure the MSE of each function over the training period and rank according to performance.

3. Select the function with the lowest MSE and calculate its MSE over the selection period. Save it as the initial best forecast function.

4. Select two functions at random, using weights attaching higher probability to more highly ranked functions. Apply the crossover oper- ator to create a new function, which then replaces an old function, chosen using weights attaching higher probability to less highly ranked functions. Repeat this procedure 500 times to create a new generation of functions.

5. Measure the MSE of each function in the new generation over the training period. Take the best function in the training period and evaluate the MSE over the selection period. If it outperforms the previous best forecast, save it as the new best forecast function.

6. Stop if no new best function appears for 25 generations, or after 50 generations. Otherwise, return to stage 4 .

The stages above describe one trial. Each trial produces one forecast function. The results of each trial will generally differ as a result of sampling variation. For this reason it is necessary to run a number of trials and then to aggregate the results. The aggregation methods are described in the following section.

\section{RESULTS}

The benchmark results are those from the $\operatorname{GARCH}(1,1)$ and RiskMetrics models described in the Benchmark Model section, estimated over the in-sample period June 1975 to December 30, 1986. We forecast daily integrated volatility (defined in equations (9) and (10)) from these models, in and out of sample, at horizons of 1,5 , and 20 days. ${ }^{4}$

We also forecast with a genetic program whose training and selection periods coincide with the insample estimation period for the GARCH model. For each case of the genetic program we generated ten trials, each of which produced a forecast function.

\footnotetext{
4 Note that the forecasted variable at the 5-day (20-day) horizon is the integrated volatility 5 (20) days in the future. It is not the sum of the next 5 (20) days of integrated volatility.
} 


\section{Table 2}

In-Sample Comparison of Genetic Program, GARCH, and RiskMetrics ${ }^{\mathrm{TM}}$ : The Baseline Case

\begin{tabular}{|c|c|c|c|c|c|c|c|c|c|c|c|c|c|}
\hline \multirow{2}{*}{$\begin{array}{l}\text { Exchange } \\
\text { rate }\end{array}$} & \multirow[b]{2}{*}{ Horizon } & \multicolumn{4}{|c|}{ MSE } & \multicolumn{4}{|c|}{ MAE } & \multicolumn{4}{|c|}{$\mathbf{R}^{2}$} \\
\hline & & EW GP & MW GP & GARCH & $\mathrm{RM}$ & EW GP & MW GP & GARCH & $\mathrm{RM}$ & EW GP & MW GP & GARCH & $\mathrm{RM}$ \\
\hline DEM & 1 & 0.50 & 0.53 & 0.50 & 0.49 & 0.30 & 0.33 & 0.33 & 0.33 & 0.18 & 0.15 & 0.16 & 0.14 \\
\hline DEM & 5 & 0.56 & 0.59 & 0.56 & 0.52 & 0.31 & 0.34 & 0.37 & 0.34 & 0.12 & 0.11 & 0.10 & 0.10 \\
\hline DEM & 20 & 0.61 & 0.63 & 0.67 & 0.56 & 0.33 & 0.34 & 0.46 & 0.37 & 0.08 & 0.04 & 0.04 & 0.05 \\
\hline JPY & 1 & 0.56 & 0.58 & 0.60 & 0.62 & 0.32 & 0.32 & 0.38 & 0.37 & 0.22 & 0.20 & 0.14 & 0.08 \\
\hline JPY & 5 & 0.65 & 0.65 & 0.73 & 0.66 & 0.36 & 0.37 & 0.43 & 0.38 & 0.06 & 0.04 & 0.02 & 0.04 \\
\hline JPY & 20 & 0.66 & 0.67 & 0.71 & 0.69 & 0.38 & 0.39 & 0.51 & 0.40 & 0.05 & 0.03 & 0.01 & 0.02 \\
\hline
\end{tabular}

NOTE: The in-sample mean-squared error (MSE), mean absolute error (MAE), and $\mathrm{R}^{2}$ from GARCH(1,1), RiskMetrics ${ }^{\mathrm{TM}}(\mathrm{RM})$, and genetic program (GP) forecasts on DEM/USD and JPY/USD data at three forecast horizons: 1, 5, and 20 days. The GP forecast was generated using five data functions and without a penalty for complexity. In columns 3, 7, and 11 we report the forecast statistics-MSE, MAE, and $\mathrm{R}^{2}$-for the equally weighted (EW) genetic programming method. In columns 4, 8, and 12 we report the analogous statistics for the median-weighted (MW) genetic programming forecast. Columns 5, 9, and 13 contain the results for the GARCH forecast. Columns 6 , 10, and 13 contain RiskMetrics ${ }^{\mathrm{TM}}$ forecast statistics. The in-sample period was June 1975 to December 30, 1986.

\section{Figure 2}

\section{One-Day-Ahead Forecasting Functions for the DEM}

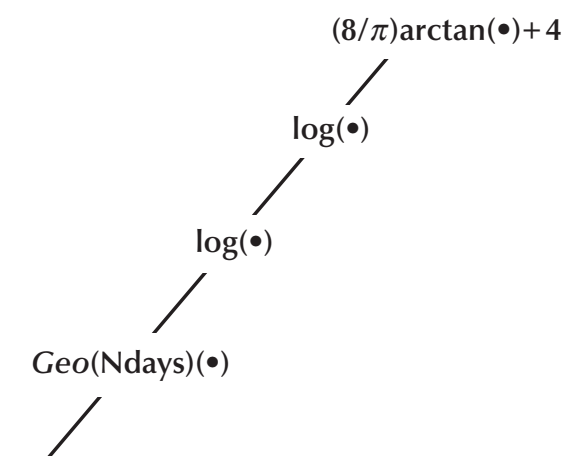

Geo(sum of squared intraday returns)(•)

$-0.4744$

The cases were distinguished by the following factors: (i) forecast horizon-1, 5, and 20 days; (ii) the number of data functions-five or eight. For each case, we generated ten rules. The forecasts from each set of ten rules were aggregated in two ways. The equally weighted forecast is the arithmetic average of the forecasts from each of the ten trials. The median-weighted forecast takes the median forecast from the set of ten forecasts at each date. We report six measures of out-of-sample forecast performance:
MSE, mean absolute error (MAE), $\mathrm{R}^{2}$, mean forecast bias, kernel estimates of the error densities, and generalized mean-squared forecast error matrix tests.

Before discussing the results, we first present a simple example of the forecasting rules produced by the genetic program. Figure 2 illustrates a oneday-ahead forecasting function for the DEM. Its out-of-sample MSE was 0.496 . The function is interpreted as follows. The number -0.4744 at the terminal node enters as the argument of geo(sum of squared intraday returns). Since the argument of geo is constrained to lie between 0.01 and 0.99 , it is set to 0.01 . The number generated by this function then enters as the argument in geo(Ndays), where Ndays refers to the "number of days to the next trading day." We caution that this example was chosen largely because of its relatively simple form; some trials generated rules that were considerably more complex, with as many as 10 levels and/or 100 nodes.

Table 2 reports in-sample results for the baseline case with five data functions. The figures for MSE for the DEM are very similar for the GARCH and equally weighted genetic program forecasts at the 1 - and 5-day horizons, but the genetic program is appreciably better at the 20-day horizon. The median-weighted forecast is generally somewhat inferior to the equally weighted forecast, but follows the same pattern over the forecast horizons relative to the GARCH model. That is, its best relative performance is at the 20-day horizon. The RiskMetrics forecasts also are generally comparable to GARCH 


\section{Table 3}

Out-of-Sample Comparison of Genetic Program, GARCH, and RiskMetrics ${ }^{\mathrm{TM}}$ : The Baseline Case

\begin{tabular}{|c|c|c|c|c|c|c|c|c|c|c|c|c|c|}
\hline \multirow{2}{*}{$\begin{array}{l}\text { Exchange } \\
\text { rate }\end{array}$} & \multirow[b]{2}{*}{ Horizon } & \multicolumn{4}{|c|}{ MSE } & \multicolumn{4}{|c|}{ MAE } & \multicolumn{4}{|c|}{$\mathbf{R}^{2}$} \\
\hline & & EW GP & MW GP & GARCH & $\mathrm{RM}$ & EW GP & MW GP & GARCH & $\mathrm{RM}$ & EW GP & MW GP & GARCH & $\mathrm{RM}$ \\
\hline DEM & 1 & 0.35 & 0.38 & 0.33 & 0.32 & 0.30 & 0.34 & 0.33 & 0.32 & 0.09 & 0.08 & 0.12 & 0.10 \\
\hline DEM & 5 & 0.38 & 0.42 & 0.36 & 0.34 & 0.31 & 0.35 & 0.35 & 0.33 & 0.06 & 0.06 & 0.08 & 0.07 \\
\hline DEM & 20 & 0.41 & 0.42 & 0.44 & 0.37 & 0.31 & 0.31 & 0.43 & 0.35 & 0.01 & 0.01 & 0.02 & 0.02 \\
\hline JPY & 1 & 1.35 & 1.35 & 1.29 & 1.33 & 0.42 & 0.44 & 0.47 & 0.47 & 0.14 & 0.13 & 0.16 & 0.11 \\
\hline JPY & 5 & 1.48 & 1.48 & 1.56 & 1.44 & 0.43 & 0.45 & 0.52 & 0.49 & 0.03 & 0.02 & 0.04 & 0.06 \\
\hline JPY & 20 & 1.48 & 1.48 & 1.43 & 1.46 & 0.45 & 0.46 & 0.55 & 0.51 & 0.02 & 0.02 & 0.05 & 0.05 \\
\hline
\end{tabular}

NOTE: The out-of-sample MSE, MAE, and $\mathrm{R}^{2}$ from $\operatorname{GARCH}(1,1)$, RiskMetrics ${ }^{\mathrm{TM}}(\mathrm{RM})$, and genetic program (GP) forecasts on DEM/USD and JPY/USD data at three forecast horizons: 1, 5, and 20 days. The GP forecast was generated using five data functions and without a penalty for complexity. The out-of-sample period was December 31, 1986, to September 21, 1999. See the notes to Table 2 for column definitions.

\section{Table 4}

\section{Out-of-Sample Results Using the Data Functions Geo, Mem, and Arch5}

\begin{tabular}{|c|c|c|c|c|c|c|c|c|c|c|c|c|c|}
\hline \multirow{2}{*}{$\begin{array}{l}\text { Exchange } \\
\text { rate }\end{array}$} & \multirow[b]{2}{*}{ Horizon } & \multicolumn{4}{|c|}{ MSE } & \multicolumn{4}{|c|}{ MAE } & \multicolumn{4}{|c|}{$\mathbf{R}^{2}$} \\
\hline & & EW GP & MW GP & GARCH & $\mathrm{RM}$ & EW GP & MW GP & GARCH & $\mathrm{RM}$ & EW GP & MW GP & GARCH & $\mathrm{RM}$ \\
\hline DEM & 1 & 0.37 & 0.44 & 0.33 & 0.32 & 0.29 & 0.37 & 0.33 & 0.32 & 0.12 & 0.05 & 0.12 & 0.10 \\
\hline DEM & 5 & 0.36 & 0.37 & 0.36 & 0.34 & 0.30 & 0.30 & 0.35 & 0.33 & 0.05 & 0.04 & 0.08 & 0.07 \\
\hline DEM & 20 & 0.38 & 0.38 & 0.44 & 0.37 & 0.30 & 0.30 & 0.43 & 0.35 & 0.01 & 0.01 & 0.02 & 0.02 \\
\hline JPY & 1 & 1.27 & 1.31 & 1.29 & 1.33 & 0.43 & 0.44 & 0.47 & 0.47 & 0.18 & 0.15 & 0.16 & 0.11 \\
\hline JPY & 5 & 1.45 & 1.46 & 1.56 & 1.44 & 0.46 & 0.46 & 0.52 & 0.49 & 0.04 & 0.03 & 0.04 & 0.06 \\
\hline JPY & 20 & 1.49 & 1.62 & 1.43 & 1.46 & 0.44 & 0.50 & 0.55 & 0.51 & 0.04 & 0.00 & 0.05 & 0.05 \\
\hline
\end{tabular}

NOTE: The out-of-sample MSE, MAE, and $\mathrm{R}^{2}$ from GARCH(1,1), RiskMetrics ${ }^{\mathrm{TM}}$ (RM), and genetic program (GP) forecasts on DEM/USD and JPY/USD data at three forecast horizons: 1, 5, and 20 days. The GP forecast was generated using eight data functions including geo, mem, and arch5 (for descriptions see equations (6) through (8) in the text) and without a penalty for complexity. The out-of-sample period was December 31, 1986, to September 21, 1999. See the notes to Table 2 for column definitions.

forecasts at 1- and 5-day horizons, but a bit better at longer horizons. For the JPY, the genetic program produces equally weighted MSE figures that are in all cases lower than for the GARCH and RiskMetrics models. Similarly, the equally weighted genetic programming rules have higher $\mathrm{R}^{2} \mathrm{~S}$ over each horizon than the GARCH and RiskMetrics models. This result is not especially surprising given the flexibility of the nonparametric procedure and its known tendency to overfit in-sample.

Table 3 presents a more interesting comparison-out-of-sample performance over the period
December 31, 1986, through September 21, 1999. The equally weighted genetic program MSE figures are usually slightly larger than those of the GARCH and RiskMetrics forecasts at all horizons for both currencies. Similarly, the genetic programming $R^{2} S$ are typically slightly smaller than those of the GARCH/RiskMetrics forecasts. However, the equally weighted genetic program has a lower MAE than do the GARCH/RiskMetrics models at all horizons and for both currencies.

Table 4 reports the out-of-sample performance of the genetic program forecasts using the augmented 


\section{Figure 3}

\section{1-Day DEM Forecast Error Densities}

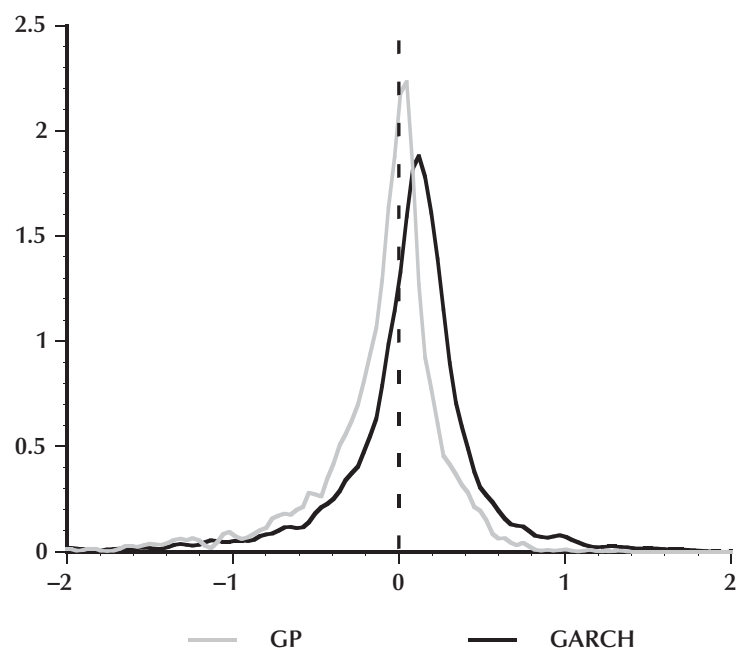

1-Day JPY Forecast Error Densities

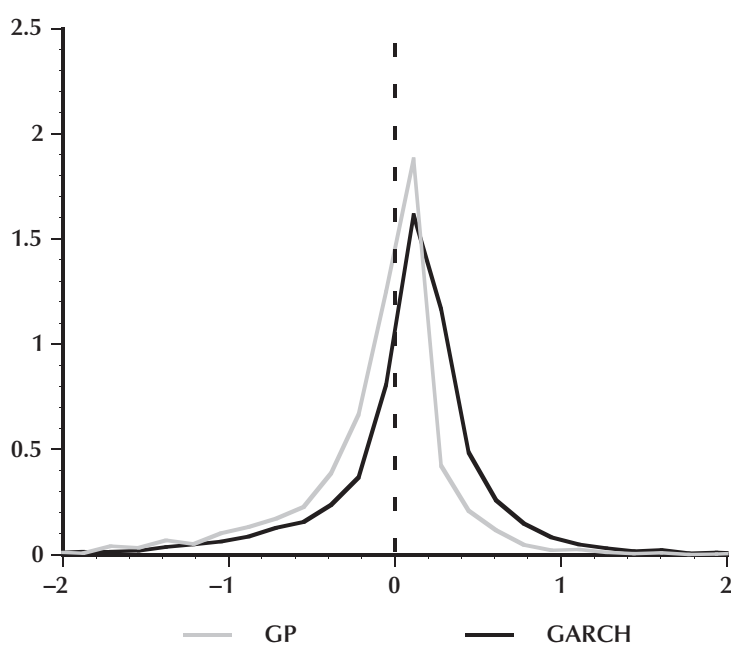

NOTE: The kernel estimates of the densities of the 1-day forecast errors (forecast minus realized volatility) for the DEM and JPY for genetic program and GARCH $(1,1)$ model over the out-of-sample period, December 31, 1986, through September 21, 1999. The dotted vertical line denotes zero.

set of data functions, which include geo, mem, and arch5. For ease of comparison Table 4 repeats the out-of-sample figures for the GARCH model. The MSE and $\mathrm{R}^{2}$ statistics from this table are more equivocal than those from Table 3. The equally weighted genetic program MSE for the DEM cases are slightly larger than those of the GARCH and RiskMetrics forecasts at the 1 - and 5-day horizons, but the genetic program performs somewhat better than GARCH at the 20-day horizon. This performance is not, however, reflected in the $\mathrm{R}^{2}$, for which the GARCH/ RiskMetrics models are better at longer horizons. For the JPY the situation is reversed; the equally weighted genetic programming MSE is lower than the GARCH/RiskMetrics figures at the 1-day horizon but larger at the 20-day horizon. The equally weighted genetic program also has a slight edge in $\mathrm{R}^{2}$ at the 1-day horizon. The figures for the MAE of the genetic program are not very different from Table 3 and are still substantially better than those of the GARCH/RiskMetrics predictions.

To summarize: With MSE as the performance criterion, neither the genetic programming procedure nor the GARCH/RiskMetrics model is clearly superior. The GARCH/RiskMetrics models do achieve slightly higher $\mathrm{R}^{2} \mathrm{~s}$ at longer horizons but the MAE criterion clearly prefers the genetic programming forecasts. In both tables, there is some tendency for the median-weighted genetic programming forecast to perform less well than its equally weighted counterpart. The out-of-sample RiskMetrics forecasts are usually marginally better than those of the estimated GARCH model by MSE and MAE criteria but marginally worse when judged by $\mathrm{R}^{2}$.

Comparing the genetic programming results in Table 4 with those of Table 3 shows that expanding the set of data functions leads to only a marginal improvement in the performance of the genetic program. Therefore further results will concentrate on out-of-sample forecasts in the baseline genetic programming case presented in Table 3, where only five data functions were used. We present kernel estimates of the densities of out-of-sample forecast errors at the various horizons in Figures 3 through 5.5

The most striking feature to emerge from these figures is the apparent bias in the GARCH forecasts when compared with their genetic program counterparts. At all forecast horizons and for both currencies, there is a positive shift in the error distributions of the GARCH forecasts that move the modes of the forecast densities away from zero. However, the relative magnitude of the bias in the mode does not carry over to the mean. Table 5 shows that, though both forecasts are biased in the mean, the magnitude of the bias is considerably greater for the genetic program. Tests for the bias-carried out with a Newey-West correction for serial correlation-show

\footnotetext{
5 We choose to graph the density of the GARCH errors because the density of the RiskMetrics errors will have a mean of approximately zero by construction.
} 


\section{Figure 4}

\section{5-Day DEM Forecast Error Densities}

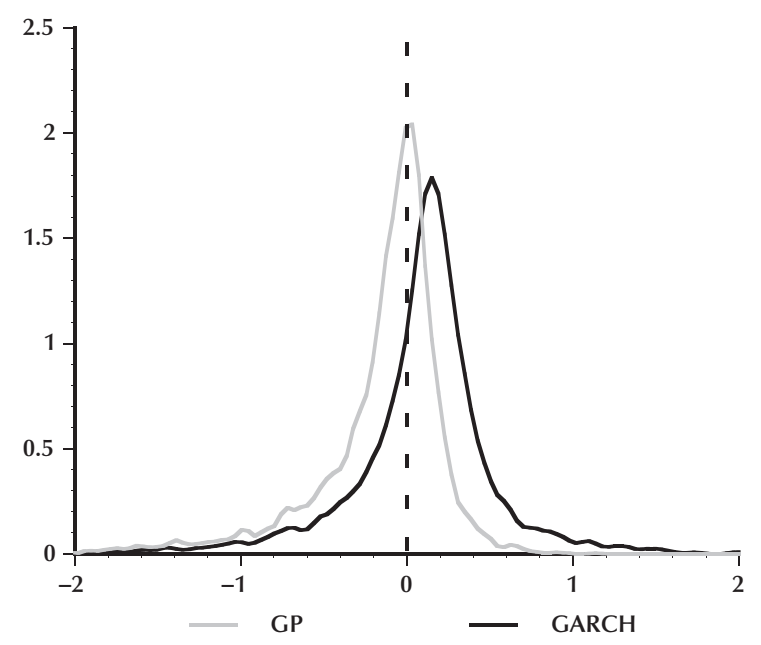

\section{5-Day JPY Forecast Error Densities}

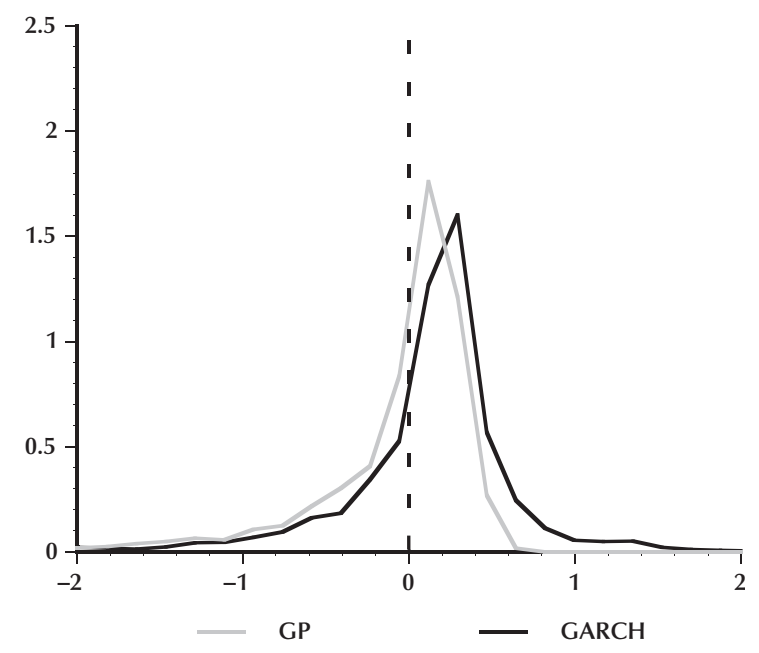

NOTE: The kernel estimates of the densities of the 5-day forecast errors (forecast minus realized volatility) for the DEM and JPY for genetic program and $\operatorname{GARCH}(1,1)$ model over the out-of-sample period, December 31, 1986, through September 21, 1999. The dotted vertical line denotes zero.

that all the forecasts are biased in a statistically significant way (Newey and West, 1987, 1994). The evidence from Figures 3 through 5 -that the modes of the genetic programming error distribution are closer to zero than those of the GARCH modelindicates that the bias in the genetic programming forecasts is being substantially influenced by a small number of negative outliers.

\section{Figure 5}

\section{0-Day DEM Forecast Error Densities}

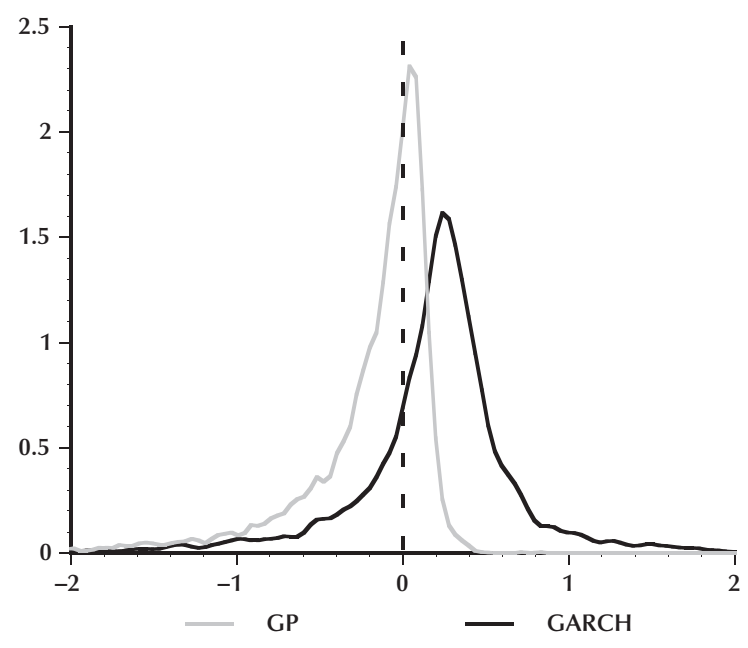

20-Day JPY Forecast Error Densities

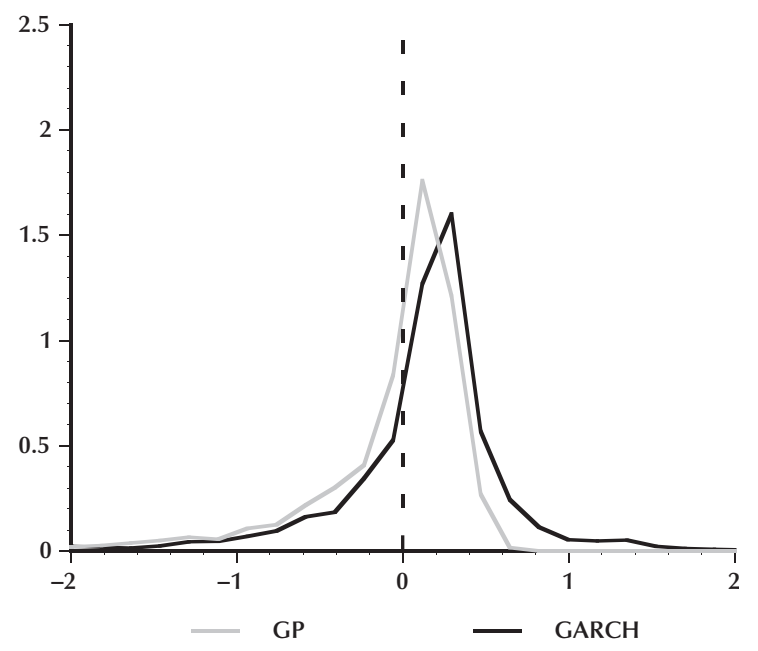

NOTE: The kernel estimates of the densities of the 20-day forecast errors (forecast minus realized volatility) for the DEM and JPY for genetic program and GARCH $(1,1)$ model over the out-of-sample period, December 31, 1986, through September 21, 1999. The dotted vertical line denotes zero.

The MSE and $\mathrm{R}^{2}$ evidence presented so far fails to indicate a clear preference for any of the four sets of forecasts. The best model varies by forecast horizon and by forecast evaluation criterion. This confused state of affairs leaves one wondering whether these disparate results can be reconciled to produce an unambiguous ranking of the two methodologies. One method by which multi-horizon 


\section{Table 5}

\section{Tests for Mean Forecast Bias}

\begin{tabular}{|c|c|c|c|c|c|c|c|c|c|c|c|c|c|c|}
\hline \multirow[b]{2}{*}{$\begin{array}{l}\text { Exchange } \\
\text { rate }\end{array}$} & \multirow[b]{2}{*}{ Horizon } & \multirow[b]{2}{*}{$\underset{\sigma^{2}}{\text { Mean }}$} & \multicolumn{3}{|c|}{$\begin{array}{c}\text { Equally weighted } \\
\text { GP }\end{array}$} & \multicolumn{3}{|c|}{$\begin{array}{c}\text { Median-weighted } \\
\text { GP }\end{array}$} & \multicolumn{3}{|c|}{ GARCH } & \multicolumn{3}{|c|}{$\mathbf{R M}$} \\
\hline & & & $\begin{array}{c}\text { Predicted } \\
\sigma^{2}\end{array}$ & Bias & $\begin{array}{l}\text { Bias } \\
p \text { value }\end{array}$ & $\begin{array}{c}\text { Predicted } \\
\sigma^{2}\end{array}$ & Bias & $\begin{array}{c}\text { Bias } \\
\mathrm{p} \text { value }\end{array}$ & $\begin{array}{c}\text { Predicted } \\
\sigma^{2}\end{array}$ & Bias & $\begin{array}{l}\text { Bias } \\
p \text { value }\end{array}$ & $\begin{array}{c}\text { Predicted } \\
\sigma^{2}\end{array}$ & Bias & $\begin{array}{l}\text { Bias } \\
\text { p value }\end{array}$ \\
\hline DEM & 1 & 0.43 & 0.29 & -0.15 & 0.00 & 0.24 & -0.19 & 0.00 & 0.46 & 0.03 & 0.00 & 0.43 & 0.00 & 0.92 \\
\hline DEM & 5 & 0.43 & 0.23 & -0.20 & 0.00 & 0.16 & -0.27 & 0.00 & 0.49 & 0.05 & 0.00 & 0.43 & 0.00 & 0.92 \\
\hline DEM & 20 & 0.43 & 0.19 & -0.24 & 0.00 & 0.18 & -0.25 & 0.00 & 0.59 & 0.16 & 0.00 & 0.43 & 0.00 & 0.92 \\
\hline JPY & 1 & 0.56 & 0.33 & -0.22 & 0.00 & 0.32 & -0.24 & 0.00 & 0.57 & 0.02 & 0.06 & 0.55 & 0.00 & 0.88 \\
\hline JPY & 5 & 0.56 & 0.37 & -0.19 & 0.00 & 0.41 & -0.15 & 0.00 & 0.59 & 0.04 & 0.07 & 0.55 & -0.01 & 0.87 \\
\hline JPY & 20 & 0.56 & 0.42 & -0.14 & 0.00 & 0.44 & -0.12 & 0.01 & 0.65 & 0.09 & 0.02 & 0.55 & -0.01 & 0.89 \\
\hline
\end{tabular}

NOTE: In column 3, mean volatility is the mean daily integrated volatility over the out-of-sample period December 31 , 1986, through September 21, 1999. Columns 4, 5, and 6 report the following statistics for the equally weighted genetic programming forecasts over the same period: mean forecast of integrated volatility, the bias in the forecast (predicted volatility minus realized volatility), and the $p$ value for the test that the mean bias is zero. Columns 7 through 9 report the statistics for the median-weighted genetic programming forecasts, and columns 10 through 12 report the analogous results for $\mathrm{GARCH}$ forecasts. The RiskMetrics ${ }^{\mathrm{TM}}$ statistics are in columns 13 through 15. The genetic program forecasts are based on the five-function model described in Table 3. The $p$ values are computed with Newey-West corrections for heteroskedasticity and serial correlation. The lag length was selected by the Newey and West (1994) procedure.

\section{Table 6}

\section{Test of Generalized Method of Second Forecast Error Moment Domination}

\begin{tabular}{cccccc} 
& \multicolumn{5}{c}{ Eigenvalues } \\
\cline { 2 - 6 } DEM & GARCH-EW GP & GARCH-MW GP & RM-EW GP & RM-MW GP & GARCH-RM \\
\hline & -0.090 & -0.148 & -0.021 & -0.037 & -0.017 \\
0.012 & -0.003 & 0.003 & -0.138 & 0.086 \\
0.082 & 0.079 & -0.084 & -0.002 & 0.036 \\
JPY & -0.369 & -0.359 & -0.028 & -0.035 & -0.295 \\
& 0.145 & 0.127 & 0.055 & 0.059 & 0.200 \\
& 0.199 & 0.203 & -0.101 & -0.102 & 0.144
\end{tabular}

NOTE: Table 6 provides sets of eigenvalues for the test of generalized method of second forecast error moment criterion. The first model dominates the second model if all the eigenvalues in a set are nonpositive and at least one is negative. GARCH-EW GP denotes the GARCH model versus the equally weighted genetic programming forecasts for the baseline case, as in Table 3. GARCH-MW GP denotes the GARCH model versus the median-weighted genetic programming forecasts for the baseline case, as in Table 3. RM-EW GP denotes the RiskMetrics ${ }^{\mathrm{TM}}$ model versus the equally weighted genetic programming forecasts for the baseline case. RM-MW GP denotes the RiskMetrics ${ }^{\mathrm{TM}}$ model versus the median-weighted genetic programming forecasts for the baseline case. GARCH-RM denotes the GARCH model versus RiskMetrics ${ }^{\mathrm{TM}}$ forecasts.

forecasts from two sources can be aggregated and compared is the generalized forecast error second moment (GFESM) method proposed by Clements and Hendry (1993). Unfortunately, this method has some drawbacks. For example, the GFESM can prefer model 1 to model 2 based on forecasts from horizon 1 to horizon $h$, even if model 2's forecasts dominate at every forecast horizon up to $h$. To remedy the perceived weaknesses in the GFESM, Newbold, Harvey, and Leybourne (1999) proposed the generalized 
mean-squared forecast error matrix (GMSFEM) criterion. This procedure prefers forecasting method 1 to method 2 if the magnitude of all linear combinations of forecast errors is at least as small under method 1 as method 2 .

To explain the GMSFEM more fully, let us introduce some notation. The one-by-three vector of 1 - 5-, and 20-day GARCH forecast errors at time $t$ is $e_{t}^{G A R C H}=\left\{e_{t, 1}^{G A R C H}, e_{t, 5}^{G A R C H}, e_{t, 20}^{G A R C H}\right\}$, and the second moment matrix of these forecast errors is $\Phi^{G A R C H}=$ $E\left(e_{t}^{G A R C H} e_{t}^{G A R C H \prime}\right)$. The RiskMetrics and genetic programming variables are defined analogously. The GMSFEM says that the GARCH model is preferred to the genetic programming forecasts if every linear combination of GARCH forecast errors is at least as small as every linear combination of genetic programming forecast errors. That is, if

$$
d^{\prime}\left(\Phi^{G A R C H}-\Phi^{G P}\right) d \leq 0 \quad \text { for all vectors } d \neq 0.6
$$

This condition is met if every eigenvalue of the matrix $\left(\Phi^{G A R C H}-\Phi^{G P}\right)$ is nonpositive and at least one is negative. Clearly, the criterion prefers the genetic programming forecast if every eigenvalue is nonnegative and at least one is positive.

Table 6 shows five sets of eigenvalues from the $\left(\Phi^{G A R C H}-\Phi^{G P}\right)$ matrix, using both the equally weighted and median-weighted genetic program forecasts, for both exchange rates. It confirms the previous results. The only case in which there are all negative (or positive) eigenvalues is the comparison of the RiskMetrics forecast to the medianweighted genetic programming forecast. In that case, all the eigenvalues are negative, indicating that the RiskMetrics forecasts dominate the medianweighted genetic programming forecasts under the GMSFEM criterion. In every other set of eigenvalues there are both positive and negative values. Neither GARCH/RiskMetrics forecasts nor genetic programming forecasts dominate the other under the GMSFEM criterion.

\section{DISCUSSION AND CONCLUSION}

We choose to use the problem of forecasting conditional volatility in the foreign exchange market to illustrate the strengths and weaknesses of genetic programming because it is a challenging problem with a well-accepted benchmark solution, the GARCH $(1,1)$ model. The genetic program did reasonably well in forecasting out-of-sample volatility.
While the genetic programming rules did not usually match the GARCH $(1,1)$ or RiskMetrics models' MSE or $\mathrm{R}^{2}$, its performance on those measures was generally close. But the genetic program did consistently outperform the GARCH model on MAE and modal error bias at all horizons. The genetic programming solutions appeared to suffer from some in-sample overfitting, which was not mitigated, in this case, by an ad hoc penalty for rule complexity. Our results suggest some interesting issues for further investigation. The superiority of the genetic program according to the MAE criterion is perhaps surprising given that we used MSE as the fitness criterion. This raises the possibility that further improvement in the forecasting performance of the genetic program relative to the GARCH model could be achieved by using MAE as the fitness criterion. Also, given that increasing the frequency of intraday observations has been shown to improve the accuracy of forecasts based on the GARCH model (Andersen et al., 2001), it is important to discover whether the results of this investigation survive in that context.

\section{REFERENCES}

Andersen, Torben and Bollerslev, Tim. "Answering the Skeptics: Yes, Standard Volatility Models Do Provide Accurate Forecasts." International Economic Review, 1998, 39(4), pp. 885-905.

; Diebold, Francis and Labys,

Paul. "Modeling and Forecasting Realized Volatility." Working Paper W8160, National Bureau of Economic Research, 2001.

Baillie, Richard and Bollerslev, Tim. "The Message in Daily Exchange Rates: A Conditional-Variance Tale.” Journal of Business and Economic Statistics, July 1989, 7(3), pp. 297-305.

and "Intra-Day and Inter-Market Volatility in Foreign Exchange Rates.” Review of Economic Studies, May 1991, 58(3), pp. 565-85.

Bollerslev, Tim. "Generalized Autoregressive Conditional Heteroskedasticity." Journal of Econometrics, April 1986, 31(3), pp. 307-27.

Chen, Shu-Heng, ed. Genetic Algorithms and Genetic

6 Note that the GMSFEM criterion implicitly favors models that do well in terms of MSE, rather than in terms of MAE. 
Programming in Computational Finance. New York: Kluwer, 2002 (forthcoming).

Clements, Michael P. and Hendry, David F. "On the Limitations of Comparing Mean Square Forecast Errors.” Journal of Forecasting, December 1993, 12(8), pp. 617-76.

Engle, Robert F. "Autoregressive Conditional Heteroskedasticity with Estimates of the Variance of U.K. Inflation." Econometrica, July 1982, 50(4), pp. 987-1007.

Holland, John. Adaptation in Natural and Artificial Systems. Ann Arbor, MI: University of Michigan Press, 1975.

J.P. Morgan/Reuters. RiskMetrics ${ }^{T M}$ Technical Document, Part II: Statistics of Financial Market Returns. Fourth Edition. New York: 1996.

Jorion, Philippe. "Predicting Volatility in the Foreign Exchange Market." Journal of Finance, June 1995, 50(2), pp. 507-28.

Koza, John R. Genetic Programming: On the Programming of Computers by Means of Natural Selection. Cambridge, MA: MIT Press, 1992.

Neely, Christopher J. "Target Zones and Conditional Volatility: The Role of Realignments.” Journal of Empirical Finance, April 1999, 6(2), pp. 177-92.

"Risk-Adjusted, Ex Ante, Optimal, Technical Trading Rules in Equity Markets.” Working Paper WP 1999-015D, Federal Reserve Bank of St. Louis, 2001 (forthcoming in International Review of Economics and Finance).

and Weller, Paul A. "Technical Trading Rules in the European Monetary System.” Journal of International Money and Finance, June 1999, 18(3), pp. 429-58.

and "Technical Analysis and

Central Bank Intervention.” Journal of International Money and Finance, December 2001, 20(7), pp. 949-70.

and Dittmar, Robert. "Is Technical Analysis in the Foreign Exchange Market Profitable? A Genetic Programming Approach." Journal of Financial and Quantitative Analysis, December 1997, 32(4), pp. 405-26.

Newbold, Paul; Harvey, David I. and Leybourne, Stephen L. "Ranking Competing Multi-Step Forecasts," in Robert F. Engle and Halbert White, eds., Cointegration, Forecasting and Causality. Chap. 4. Oxford: Oxford University Press, 1999.

Newey, Whitney K. and West, Kenneth D. "A Simple Positive, Semi-Definite, Heteroskedasticity and Autocorrelation Consistent Covariance Matrix." Econometrica, May 1987, 55(3), pp. 703-08.

and "Automatic Lag Selection in Covariance Matrix Estimation." Review of Economic Studies, October 1994, 61(4), pp. 631-53.

West, Kenneth D. and Cho, Dongchul. "The Predictive Ability of Several Models of Exchange Rate Volatility." Journal of Econometrics, October 1995, 69(2), pp. 367-91. 